\title{
Effect of heat treatment on the physicochemical properties of nitrogen-enriched activated carbons
}

\author{
P. Nowicki ${ }^{1}$
}

Received: 9 October 2015/Accepted: 14 January 2016/Published online: 12 February 2016

(C) The Author(s) 2016. This article is published with open access at Springerlink.com

\begin{abstract}
A series of functionalized carbonaceous adsorbents were prepared by means of reaction with urea and chemical activation of Polish brown coal. In order to obtain nitrogen groups bonded in different ways to the carbon structure, the reaction with urea was performed at two different stages of processing, with precursor or char. Additionally, the influence of annealing under nitrogen atmosphere at 600 and $800{ }^{\circ} \mathrm{C}$ or in hydrogen/nitrogen mixture at $600{ }^{\circ} \mathrm{C}$ on the textural, acid-base and thermal properties of activated carbons prepared was tested. The resulting materials were characterized by elemental analysis, low-temperature nitrogen sorption, determination of the number of surface oxygen groups as well as by thermogravimetric study in helium atmosphere. The final products were microporous nitrogen-enriched activated carbons of very well-developed surface area reaching from 1303 to $2004 \mathrm{~m}^{2} \mathrm{~g}^{-1}$, showing different content of nitrogen (from 0.5 to 2.3 mass\%) and different chemical character of the surface (from weakly acidic to intermediate acidic-basic), depending on the sequence of particular processes and variant of further thermochemical treatment. According to the results of thermal study, the nitrogen functional groups introduced into the carbon structure by the reaction with urea (especially at the char stage) show moderate thermal and chemical stability. Under the effect of high temperature, some of these groups underwent decomposition or transformation into more thermally stable nitrogen species. It was also proved that the
\end{abstract}

P. Nowicki

piotrnow@amu.edu.pl

1 Laboratory of Applied Chemistry, Faculty of Chemistry, Adam Mickiewicz University in Poznań, Umultowska 89b, 61-614 Poznan, Poland thermochemical treatment of modified activated carbons led to considerable deterioration of their textural parameters as well as caused significant changes in the acid-base character of their surface.

Keywords Brown coal - Chemical activation - Nitrogenenriched activated carbons - Physicochemical properties . Thermal study

\section{Introduction}

Modified adsorbents have been for a long time the subject of interest of research groups all over the world [1-9] mainly because of a wide spectrum of their application. They are used both in protection of the natural environment as adsorbents of toxic gases [10-14] or liquids [15, 16] and in modern industry as catalyst or catalyst supports [17-19] as well as electrode materials for electrochemical capacitors $[20,21]$. This wide range of their use is a consequence of their unique properties determined by the well-developed porous structure and the possibility of their modification with different functional groups incorporated into their structure. No wonder that much research work aimed at development of new materials of the target physicochemical properties at optimization of the already known ones is continuously going on.

Of particular interest from among modified carbon materials are those enriched in oxygen and/or nitrogen functional groups. The first attempts at developing such materials date back to the beginning of the twentieth century when the activated carbon materials were obtained by pyrolysis and activation of organic substances containing significant amounts of nitrogen, like polyamides or animal blood. It was followed by the period of intense studies on 
the obtaining of nitrogenized activated carbons and their potential use.

As follows from literature data, the materials can be obtained by many ways, but all of them are based on one of the three fundamental methods: (1) thermal processing of the precursor or activated carbon in the presence of the socalled N-reagents, so compounds containing nitrogen in their structure (e.g. ammonia [22, 23], urea [24, 25], nitrogen oxides [26], hydroxyl amine and hydrazine [27], (2) pyrolysis/activation (mainly physical) of polymers or rich in nitrogen vegetation origin precursors (e.g. polyacrylonitrile [28], polyamides [29], polyimides [30] and waste material left after soybean growing and processing [31]; and (3) impregnation of carbons with solutions of amines and imines of any order [32,33] or coating carbons with a layer of polymers containing nitrogen in their structure [34]. Depending on the type of precursor and the variant of its modification, the carbon materials obtained are characterized by different contents of nitrogen and their different types, i.e. different types of functional groups and their different position in the carbon structure.

In previous studies, nitrogen-doped activated carbons have been prepared mainly by physical activation with steam or carbon dioxide, whereas much less attention has been paid to chemical methods of activation. Therefore, the main aim of this study was to obtain nitrogen-enriched activated carbons by reaction with urea and chemical activation of brown coal and to check the influence of thermochemical treatment on their textural, acid-base and thermal properties.

\section{Experimental}

\section{Materials preparation}

The starting raw sample (S) was prepared from Polish brown coal (Konin colliery, ash $=25.3$ mass $\%$, volatile matter $=55.6$ mass $\%$, moisture $=11.3$ mass $\%$, fixed carbon $=61.4$ mass $\%)$. At the beginning, the precursor was milled and sieved to the grain size of $0.5-1 \mathrm{~mm}$ and then it was subjected to demineralization by treatment with concentrated $\mathrm{HCl}$ and $\mathrm{HF}$. After that, the demineralized coal (D) was subjected to two different modes of treatment: (1) reaction with urea followed by pyrolysis and chemical activation by $\mathrm{KOH}$ (UPA sample) and (2) pyrolysis followed by reaction with urea and chemical activation (PUA sample). The unmodified activated carbon (PA) was used as a reference.

Incorporation of nitrogen (U): $20 \mathrm{~g}$ of carbonaceous materials was impregnated with urea at the mass ratio of $1: 1$, dried at $110{ }^{\circ} \mathrm{C}$ to constant mass and then subjected to thermal treatment. The process proceeded in a glass reactor under atmospheric pressure for $3 \mathrm{~h}$, at $350{ }^{\circ} \mathrm{C}$, in air flow $\left(100 \mathrm{~mL} \mathrm{~min}^{-1}\right)$. The nitrogen-enriched materials were washed with hot distilled water in order to remove the unreacted part of urea and dried to constant mass. Nitrogen was incorporated to demineralized coal as well as to the product of its pyrolysis.

Pyrolysis $(\mathrm{P})$ was performed in a quartz tubular reactor heated by a resistance furnace at $700{ }^{\circ} \mathrm{C}$, under argon flow (flow rate of $170 \mathrm{~mL} \mathrm{~min}^{-1}$ ). The samples were heated at the rate $5{ }^{\circ} \mathrm{C} \mathrm{min}{ }^{-1}$ from room temperature to final pyrolysis temperature, maintained for $1 \mathrm{~h}$ and finally cooled down to room temperature.

Activation (A): $\mathrm{KOH}$ was directly mixed at room temperature with samples at the mass ratio of $4: 1$. After the physical mixing, the samples were heated at $700{ }^{\circ} \mathrm{C}$ under argon flow (flow rate $330 \mathrm{~mL} \mathrm{~min}^{-1}$ ) for $45 \mathrm{~min}$, in a ceramic tubular reactor heated by a resistance furnace. The obtained activated carbons were washed first with $5 \% \mathrm{HCl}$ solution and then with distilled water until free of chloride ions. The washed activated carbons were dried at $110{ }^{\circ} \mathrm{C}$ to constant mass.

Thermochemical treatment of the activated carbons: all prepared samples were subjected to annealing for $3 \mathrm{~h}$ in three variants: under nitrogen atmosphere at $600{ }^{\circ} \mathrm{C}(6)$, under nitrogen atmosphere at $800{ }^{\circ} \mathrm{C}(8)$ and in hydrogen/ nitrogen mixture at $600{ }^{\circ} \mathrm{C}(\mathrm{H})$.

\section{Analytical procedures}

Proximate analysis of the starting material (determination of moisture, ash and volatile matter) was performed according to PN-80/G-04511, PN-ISO 1171:2002 and PN-ISO 562:2000 standards, respectively. Elemental analysis of all samples under investigation was carried out using the Elementar Analysensysteme instrument, model Vario EL III.

The textural characterization of the samples was based on nitrogen adsorption-desorption isotherms measured at $-196{ }^{\circ} \mathrm{C}$ with a Quantachrome Autosorb iQ surface area analyser. Before measurements, the samples were outgassed at $150{ }^{\circ} \mathrm{C}$ for $8 \mathrm{~h}$. Surface area and pore size distribution were calculated by BET (Brunauer-EmmettTeller) and BJH (Barrett-Joyner-Halenda) methods, respectively. Average pore diameter and total pore volume were determined as well. Micropores volume was calculated using the t-plot method. The calculations of the PSDs (pore size distributions) were performed using the carbon$\mathrm{N}_{2}$ NLDFT standard slit model implemented in the SAIEUS software (Micromeritics), www.nldft.com.

The surface functional groups of acidic and basic character were determined according to the Boehm method.

Thermogravimetric analysis of investigated materials was performed on a SETSYS 12, made by Setaram. The samples $(\sim 20 \mathrm{mg}$, particle size below $0.1 \mathrm{~mm})$ were 
heated at the rate $10{ }^{\circ} \mathrm{C} \mathrm{min}^{-1}$, from room temperature to $900{ }^{\circ} \mathrm{C}$, under helium atmosphere.

\section{Results and discussion}

\section{Elemental composition of materials prepared}

According to the data presented in Table 1, demineralization removes over $97 \%$ of the mineral substance from the raw brown coal and leads to only insignificant changes in the organic components of this coal. Thermochemical treatment of the demineralized coal (irrespective of the variant) brings a substantial increase in the content of $\mathrm{C}^{\text {daf }}$, accompanied by a slight increase in the nitrogen contribution (in UPA and PUA samples) and considerable decrease in the content of the other elements. These changes are certainly related to the high temperature of the processes, which is responsible for breaking of the least stable chemical bonds present in the material, removal of heteroatoms in the form of simple gas or liquid compounds and consequently for the ordering of carbonaceous structure. The increase in nitrogen content observed for UPA and PUA samples is most probably related to the nitrogen building into the carbon structure during the reaction with urea, in the form of amines, amides, imines, lactams or nitriles [35, 36]. Nevertheless, under the effect of high temperature (during pyrolysis or activation processes), a considerable part of these groups underwent decomposition or transformation to more thermally stable nitrogen species (e.g. N-5, N-6 or N-Q $[35,36]$ ) and consequently the amount of nitrogen in the products of activation is not too

Table 1 Elemental composition of the precursor, demineralized coal and activated carbons (mass\%)

\begin{tabular}{lrlllll}
\hline Sample & Ash & $\mathrm{C}^{\text {daf } *}$ & $\mathrm{H}^{\text {daf }}$ & $\mathrm{N}^{\text {daf }}$ & $\mathrm{S}^{\text {daf }}$ & $\mathrm{O}^{\text {daf } * *}$ \\
\hline S & 25.3 & 61.4 & 4.8 & 0.7 & 2.0 & 31.1 \\
D & 0.7 & 62.1 & 4.5 & 0.7 & 1.9 & 30.8 \\
UPA & 1.2 & 88.4 & 0.9 & 2.3 & 0.2 & 8.2 \\
UPA6 & 1.6 & 89.5 & 1.0 & 2.2 & 0.2 & 7.1 \\
UPA8 & 1.8 & 92.5 & 1.1 & 2.0 & 0.2 & 4.2 \\
UPAH & 1.3 & 93.9 & 1.0 & 1.8 & 0.1 & 3.2 \\
PUA & 1.6 & 89.6 & 0.8 & 1.2 & 0.1 & 8.3 \\
PUA6 & 2.0 & 90.7 & 0.7 & 1.1 & 0.1 & 7.4 \\
PUA8 & 2.3 & 91.2 & 1.0 & 1.0 & 0.1 & 6.7 \\
PUAH & 1.5 & 94.8 & 0.8 & 0.8 & 0.1 & 3.5 \\
PA & 1.2 & 92.3 & 0.5 & 0.6 & 0.0 & 6.6 \\
PA6 & 1.4 & 94.2 & 0.4 & 0.6 & 0.0 & 4.8 \\
PA8 & 1.7 & 96.5 & 0.5 & 0.6 & 0.0 & 2.4 \\
PAH & 1.1 & 96.9 & 0.6 & 0.5 & 0.0 & 2.0 \\
\hline
\end{tabular}

* Dry-ash-free basis; ** determined by difference high, especially for sample PUA, modified with nitrogen just before the activation process. High-temperature treatment of the demineralized coal causes also a significant increase in the ash content, as evidenced by the fact that the activation products are characterized by almost twice higher content of mineral substances than the starting material.

As follows from further analysis of the data collected in Table 1, each of the post-activation treatments performed led to significant changes in the content of mineral substance and in the contribution of particular elements $(\mathrm{C}, \mathrm{H}$, $\mathrm{N}, \mathrm{S}, \mathrm{O}$ ) in the activated carbons structure. The character of changes depends significantly on the variant of modification and to a lower extent on the type of material subjected to the thermochemical treatment. First of all, we can observe a significant increase in the contribution of carbon, accompanied by a simultaneous decrease in the oxygen content as well as slight changes in the nitrogen and hydrogen content. These changes are more pronounced for UPAH, PUAH and PAH samples, annealed at $600{ }^{\circ} \mathrm{C}$ in nitrogen/hydrogen mixture. Exposure of the activated carbon to $\mathrm{N}_{2} / \mathrm{H}_{2}$ mixture leads mainly to a considerable decrease in the content of oxygen (over twofold) and nitrogen. Annealing of the activated carbons under a stream of nitrogen (even at $800{ }^{\circ} \mathrm{C}$ ) causes smaller changes in the elemental composition, than heat treatment in $\mathrm{N}_{2} / \mathrm{H}_{2}$ mixture. Similarly as for UPAH, PUAH and PAH samples, we can observe a decrease in the oxygen content, especially for UPA8, PUA8 and PA8 samples, but the intensity of these changes is much lower. It should be also noted that the nitrogen content decreases to a much smaller extent. This fact indicates a relatively high thermal stability of the nitrogen groups present in the activated carbons structure. On the other hand, a significant decrease in the nitrogen content observed for UPAH and PUAH samples (heated in a stream of $\mathrm{N}_{2} / \mathrm{H}_{2}$ ) suggests weak chemical bonds of the introduced nitrogen functional groups. However, verification of this supposition needs further studies.

\section{Acid-base properties of materials prepared}

To establish the acid-base properties of the activated carbon samples obtained, the contents of functional groups of acidic or basic character were determined. As follows from the data presented in Table 2, the carbonaceous materials differ significantly in the number and type of acidic and basic functional groups. The content of the surface functional groups depends to a high extent on the sequence of processes to which the demineralized coal was subjected (reaction with urea, pyrolysis, activation). The greatest amount of surface groups (from among the materials not subjected to post-activation treatment) was determined in 
Table 2 Content of acidic and basic surface functional groups for the activated carbons

\begin{tabular}{llll}
\hline Sample & $\begin{array}{l}\text { Acidic groups/ } \\
\mathrm{mmol} \mathrm{g}^{-1}\end{array}$ & $\begin{array}{l}\text { Basic groups/ } \\
\mathrm{mmol} \mathrm{g}^{-1}\end{array}$ & $\begin{array}{l}\text { Total content/ } \\
\mathrm{mmol} \mathrm{g}^{-1}\end{array}$ \\
\hline UPA & 1.72 & 0.81 & 2.53 \\
UPA6 & 1.28 & 0.69 & 1.97 \\
UPA8 & 0.91 & 0.75 & 1.66 \\
UPAH & 1.36 & 0.65 & 2.01 \\
PUA & 1.45 & 0.68 & 2.03 \\
PUA6 & 1.09 & 0.51 & 1.60 \\
PUA8 & 0.81 & 0.64 & 1.45 \\
PUAH & 1.21 & 0.57 & 1.78 \\
PA & 1.24 & 0.70 & 1.94 \\
PA6 & 1.05 & 0.42 & 1.47 \\
PA8 & 0.74 & 0.49 & 1.23 \\
PAH & 1.12 & 0.43 & 1.45 \\
\hline
\end{tabular}

sample UPA subjected to the reaction with urea at the stage of precursor, while the lowest was in the sample unmodified with nitrogen (PA). Moreover, for all the activated carbons not subjected to heat treatment, a great (almost twofold) predominance of acidic groups was observed. The amount of acidic and basic moieties (similarly as the total content of functional groups) to a high degree depends on the stage at which the sample was subjected to reaction with urea.

According to the data presented in Table 2, annealing of the activated carbons under nitrogen or nitrogen/hydrogen mixture results in substantial changes in their acid-base properties. Irrespective of the variant of thermal treatment, we observed mainly a significant decrease in the acidic group content. This process occurs with the highest efficiency for the samples heated at $800{ }^{\circ} \mathrm{C}$ in nitrogen atmosphere, especially for UPA8 sample, which contains almost twice less acidic species on their surface, than UPA sample, not subjected to thermal modification. These changes are certainly related to the high temperature of the process, which contributes to the decomposition of acidic moieties. In the samples modified in $\mathrm{N}_{2} / \mathrm{H}_{2}$ mixture and nitrogen atmosphere at $600{ }^{\circ} \mathrm{C}$, the changes are similar to the former, but are less pronounced. Moreover, all of the samples subjected to heat treatment are characterized by lower content of surface groups of basic character than the corresponding unmodified activated carbons; however, the difference is not so much pronounced as for the acidic moieties.

\section{Textural parameters of materials prepared}

Textural parameters of the samples studied were determined from the low-temperature nitrogen adsorption/ desorption measurements performed on a Quantachrome Autosorb iQ surface area analyser. As follows from the results shown in Table 3, all the activated carbons prepared from brown coal have well-developed surface area and porous structure with dominant contribution of micropores. The efficiency of porous structure development depends on the sequence of the processes to which the demineralized coal was subjected. The activated carbons subjected to reaction with urea at the stage of demineralized coal (UPA) and at the stage of char (PUA) have much greater surface area than those to which nitrogen was not introduced (PA). The same tendency is observed for total pore volume as well as micropore contribution. The most probable reason for such a strong development of the porous structure of the samples enriched in nitrogen (in particular at the precursor stage) is the enhanced reactivity of the modified precursor and char towards activating agent, following from the presence of reactive nitrogen and oxygen functional groups introduced upon reaction with urea, which promote a stronger development of the porous structure.

Table 3 data clearly illustrate a considerable influence of the conditions of post-activation heat treatment on the porous structure of the activated carbons. As shown, thermal modification of UPA, PUA as well as PA samples (both in nitrogen and nitrogen/hydrogen atmosphere) leads to the deterioration of their surface area and pore volume. This phenomenon is probably a result of destruction of walls between neighbouring pores and formation of wider micropores and small mesopores. This hypothesis is confirmed by a considerable increase in the average pore diameter as well as by a simultaneous decrease in micropores contribution. The deterioration of textural parameters after thermal modification is the most pronounced for the activated carbons heated in nitrogen atmosphere at $800{ }^{\circ} \mathrm{C}$, especially for UPA8 and PUA8 samples, whose surface area is over $20 \%$ lower than for the corresponding samples UPA and PUA, not subjected to heat treatment. A similar tendency is observed for UPA6, UPAH and PUA6 samples. For the remaining activated carbons annealed at $600{ }^{\circ} \mathrm{C}$ (under $\mathrm{N}_{2}$ or $\mathrm{N}_{2} / \mathrm{H}_{2}$ atmosphere), the deterioration of surface area is lower and varies from 3 to $12 \%$.

Microporous character of the structure of the activated carbons obtained is also confirmed by the pore size distributions presented in Figs. 1-3. According to these results, the porous structure of all carbons studied mainly contains micropores and small mesopores of diameters below $30 \AA$. Analysis of the results also implies that different variants of thermochemical treatment cause different changes in the contributions of particular type pores. A decrease in the contribution of micropores of diameters about $10 \AA$ is observed accompanied by an increase in the contribution of pores of larger diameters. It is particularly 
Table 3 Textural parameters of the samples

\begin{tabular}{lllll}
\hline Sample & Surface area/ $\mathrm{m}^{2} \mathrm{~g}^{-1}$ & Total pore volume $/ \mathrm{cm}^{3} \mathrm{~g}^{-1}$ & Micropore contribution/\% & Average pore diameter/A \\
\hline UPA & 2004 & 0.98 & 95 & 19.3 \\
UPA6 & 1598 & 0.90 & 93 & 22.8 \\
UPA8 & 1547 & 0.89 & 91 & 23.0 \\
UPAH & 1640 & 0.92 & 90 & 23.4 \\
PUA & 1650 & 0.89 & 94 & 19.8 \\
PUA6 & 1352 & 0.77 & 89 & 23.9 \\
PUA8 & 1303 & 0.75 & 85 & 24.2 \\
PUAH & 1455 & 0.82 & 87 & 23.9 \\
PA & 1589 & 0.81 & 92 & 19.7 \\
PA6 & 1525 & 0.82 & 88 & 23.3 \\
PA8 & 1475 & 0.75 & 86 & 24.0 \\
PAH & 1548 & 0.84 & 90 & 23.1 \\
\hline
\end{tabular}

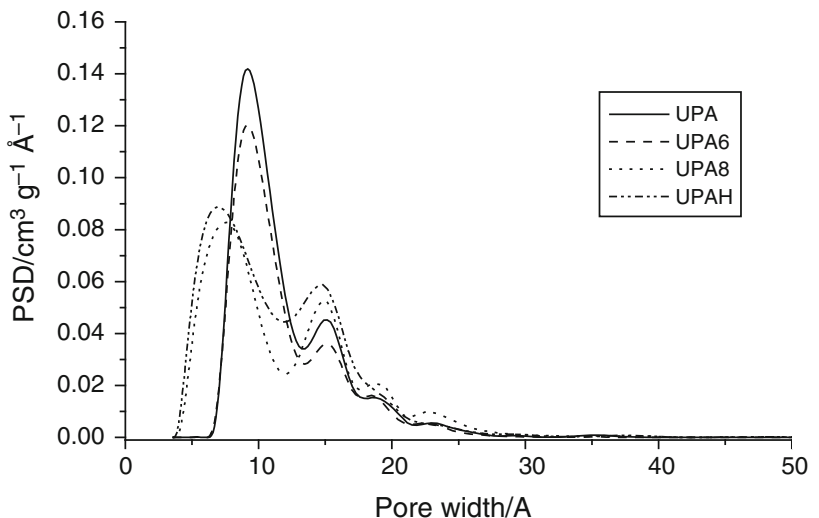

Fig. 1 Pore size distributions of activated carbon enriched in nitrogen at the precursor stage and samples obtained by its heat treatment in different conditions

well seen for samples UPA and PUA annealed under $\mathrm{N}_{2}$ at $800{ }^{\circ} \mathrm{C}$ or in $\mathrm{N}_{2} / \mathrm{H}_{2}$ atmosphere.

\section{Thermal properties of materials prepared}

Thermal properties of the samples under investigation were characterized by thermogravimetric measurements in the helium atmosphere. As follows from the character of TG and DTG curves, presented in Figs. 4-9, the activated carbons differ significantly in thermal stability. The thermal properties of the materials studied depend on the sequence of the processes to which the demineralized coal was subjected as well as on the variant of the post-activation heat treatment. The most thermally stable (among samples not subjected to post-activation treatment) is sample PA, to which nitrogen was not introduced at any stage of processing. The total mass loss for this sample is only $4 \%$ (Fig. 6). The DTG curve recorded for this sample (Fig. 9) shows four slightly marked minima: at about

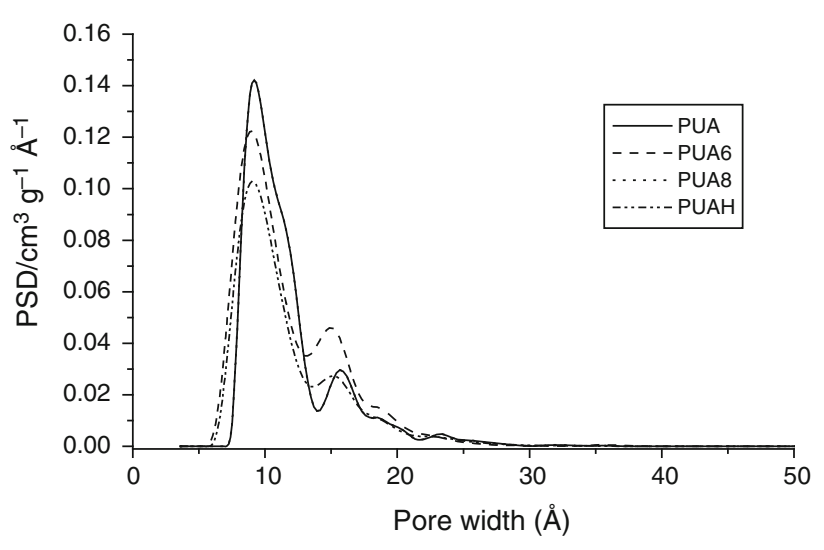

Fig. 2 Pore size distributions of activated carbon enriched in nitrogen at the char stage and samples obtained by its heat treatment in different conditions

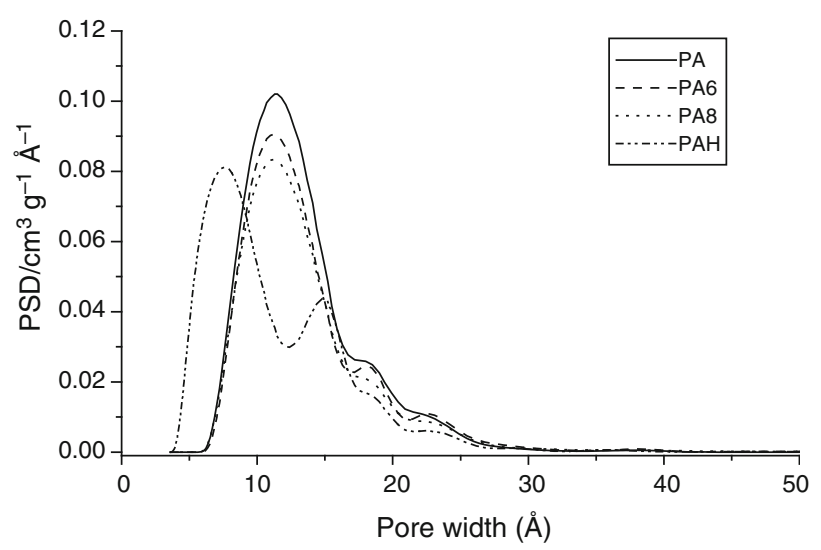

Fig. 3 Pore size distributions of unmodified activated carbon and samples obtained by its heat treatment in different conditions

50-60 ${ }^{\circ} \mathrm{C}$ corresponding to the desorption of the physically adsorbed water, at about 250 and $400{ }^{\circ} \mathrm{C}$ which can be assigned to decomposition of poorly thermally 
stable oxygen functional groups generated on the carbon surface upon the activation process (such as carboxyl and lactone groups), while the minima at $620-630{ }^{\circ} \mathrm{C}$ can be attributed to decomposition of acidic anhydrides. There is also a broad peak above $700{ }^{\circ} \mathrm{C}$ which can be related to slow decomposition of the organic components of carbon matrix.

For the nitrogen-enriched activated carbons (UPA and PUA, Figs. 4, 5, respectively), the mass loss is considerably higher and reaches up to $12-13 \%$. The DTG curves of these samples (Figs. 7, 8) also show a characteristic minimum attributed to the desorption of physisorbed water (at $60-80{ }^{\circ} \mathrm{C}$ ); however, it is much more pronounced than for sample PA, particularly for the sample subjected to the reaction with urea at the precursor stage (UPA). The presence of this minimum suggests that incorporation of significant amount of nitrogen and oxygen functional groups causes a considerable increase in the hydrophilic properties of the samples' surfaces. The DTG curves of UPA and PUA show also two very broad peaks with minima at about $270-320$ and $700-720{ }^{\circ} \mathrm{C}$. The first of them can be assigned to the decomposition of poorly thermally stable nitrogen moieties introduced upon reaction with urea (for example imines and lactams) as well as oxygen groups generated during chemical activation with $\mathrm{KOH}$. The second peak can be assigned to the decomposition of nitrogen groups in which nitrogen is built into the aromatic ring, so pyridine, pyrrole or pyridine $\mathrm{N}$-oxide.

As follows from further analysis of the data presented in Figs. 6-9, each of the post-activation treatments performed resulted in significant changes in the thermal properties of activated carbons. As a result of annealing of the activated carbons in nitrogen atmosphere (especially at $800{ }^{\circ} \mathrm{C}$ ), the thermal stability of the samples considerably increases. It is clearly evidenced by the lower mass loss during the

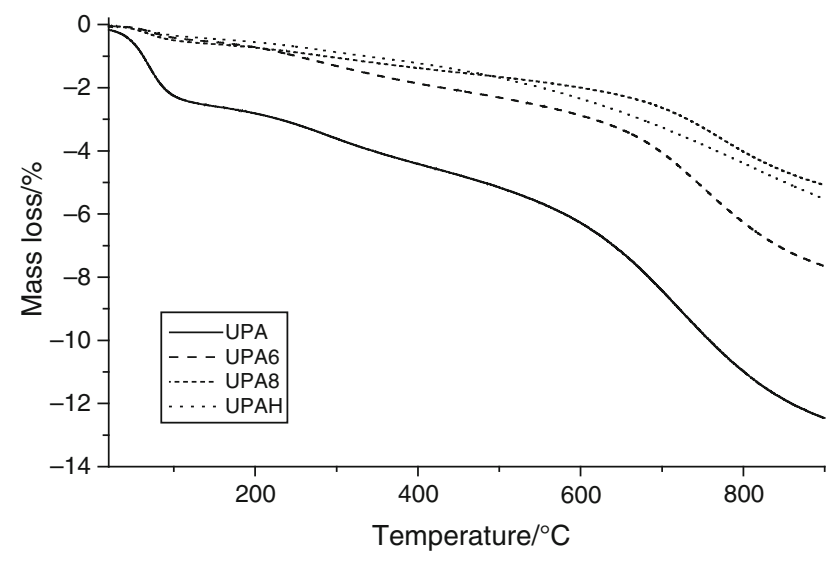

Fig. 4 TG curves of activated carbon enriched in nitrogen at the precursor stage and samples obtained by its heat treatment in different conditions

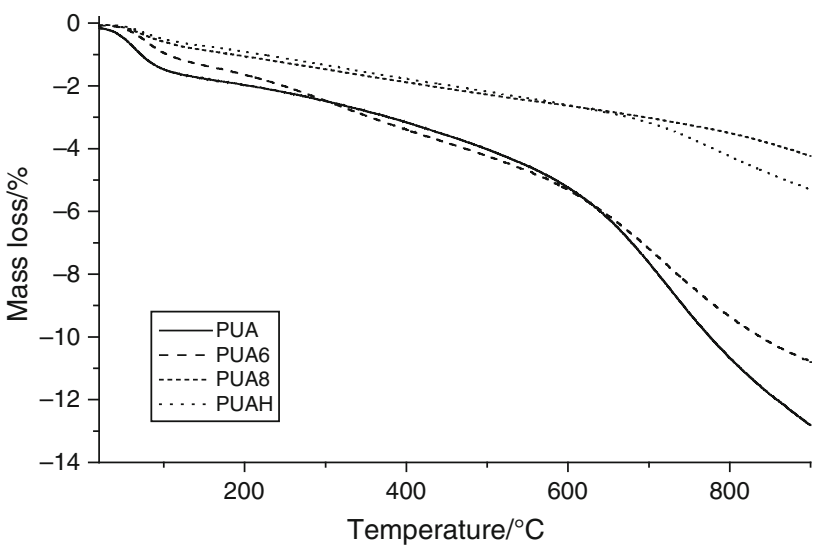

Fig. 5 TG curves of activated carbon enriched in nitrogen at the char stage and samples obtained by its heat treatment in different conditions

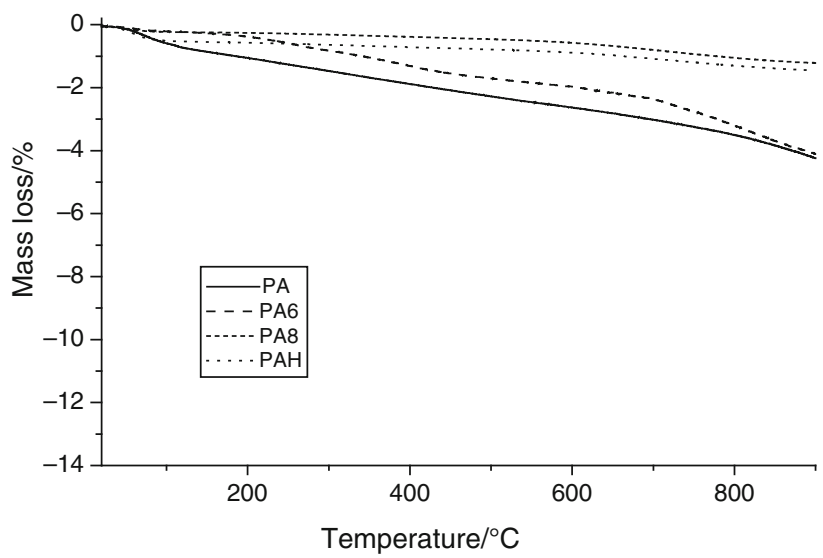

Fig. 6 TG curves of unmodified activated carbon and samples obtained by its heat treatment in different conditions

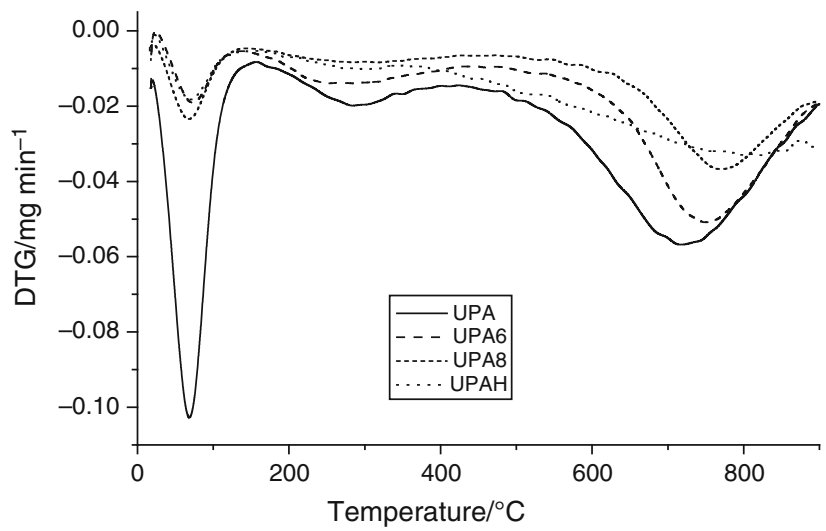

Fig. 7 DTG curves of activated carbon enriched in nitrogen at the precursor stage and samples obtained by its heat treatment in different conditions

thermogravimetric study (Figs. 4-6) as well as by the lower intensity of particular minima observed on DTG curves and their shift to higher temperatures. It is 


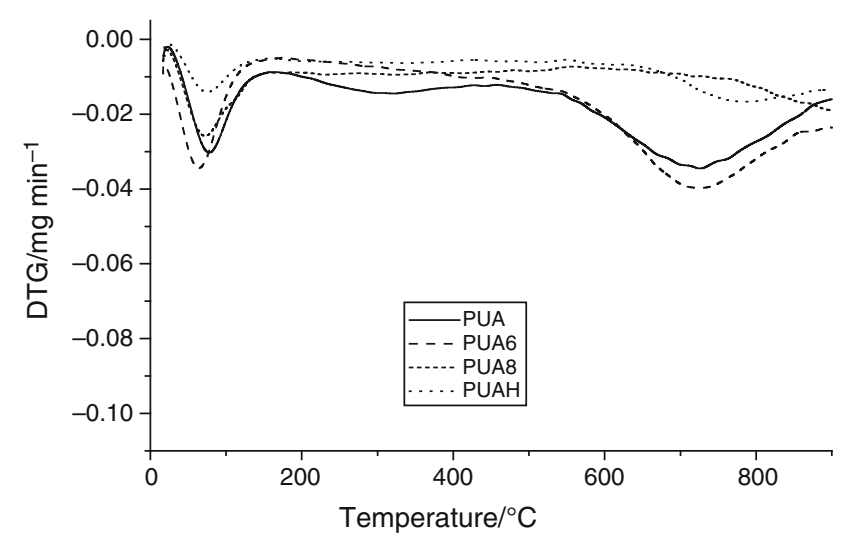

Fig. 8 DTG curves of activated carbon enriched in nitrogen at the char stage and samples obtained by its heat treatment in different conditions

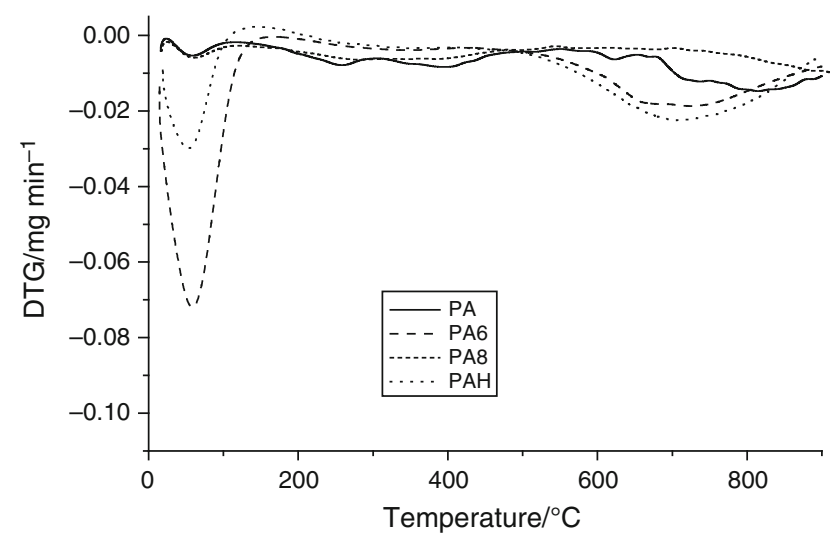

Fig. 9 DTG curves of unmodified activated carbon and samples obtained by its heat treatment in different conditions

particularly well seen for samples UPA6 and UPA8 (Fig. 7). A similar effect can be achieved by exposition of the activated carbon to a nitrogen/hydrogen mixture. However, thermal stability of so-prepared materials is intermediate between the values obtained for the samples heated in nitrogen at 600 and $800{ }^{\circ} \mathrm{C}$.

\section{Conclusions}

The above-discussed results have proved that it is possible to obtain from brown coal a large gamut of nitrogen-enriched activated carbons of very well-developed porous structure, showing different content of nitrogen and acidbase properties of the surface, depending on the sequence of particular processes and variant of thermochemical treatment.

Thermal study results have evidenced that nitrogen functional groups introduced into carbon structure by the reaction with urea show moderate thermal and chemical stability. Under the effect of high temperature and/or potassium hydroxide, a part of these groups underwent decomposition or transformation into more thermally stable nitrogen species. However, verification of this supposition needs further studies.

It has been also proved that the thermochemical treatment of nitrogen-enriched activated carbons led to considerable deterioration of their textural parameters as well as caused significant changes in the acid-base character of their surface.

Acknowledgements This work was partially supported by Ministry of Science and Higher Education of Poland (Project Iuventus Plus No. IP2012 004072).

Open Access This article is distributed under the terms of the Creative Commons Attribution 4.0 International License (http://creative commons.org/licenses/by/4.0/), which permits unrestricted use, distribution, and reproduction in any medium, provided you give appropriate credit to the original author(s) and the source, provide a link to the Creative Commons license, and indicate if changes were made.

\section{References}

1. Budarin VL, Clark JH, Gorlova AA, Boldyreva NA, Yatsimirsky VK. Chemical modification of activated carbons. J Therm Anal Calorim. 2000;62:349-52.

2. Rodríguez-Estupiñán P, Giraldo L, Moreno-Piraján JC. Modified surface chemistry of activated carbons: correlation with immersion enthalpy. J Therm Anal Calorim. 2013;114:245-51.

3. Makomaski G, Ciesińska W, Zieliński J. Thermal properties of pitch-polymer compositions and derived activated carbons. J Therm Anal Calorim. 2012;109:767-72.

4. Abdul Khalil HPS, Firoozian P, Jawaid M, Akil HM, Hassan A. Preparation of activated carbon filled epoxy nanocomposites: morphological and thermal properties. J Therm Anal Calorim. 2013;113:623-31.

5. Bylina IV, Tong S, Jia CQ. Thermal analysis of sulphur impregnated activated carbons with mercury adsorber from the vapour phase. J Therm Anal Calorim. 2009;96:91-8.

6. Shawabkeh R, Aslam Z, Hussien I. Thermochemical treatment of fly ash for synthesis of mesoporous activated carbon. J Therm Anal Calorim. 2015;122:1191-201.

7. Wiśniewska M, Chibowski S, Urban T. Impact of polyacrylamide with different contents of carboxyl groups on the chromium (III) oxide adsorption properties in aqueous solution. J Hazard Mater. 2015;283:815-23.

8. Nosal-Wiercińska A, Grochowski M, Wiśniewska M, TyszczukRotko K, Yilmaz S, Yagmur S, Saglikoglu G, Yanik S. Adsorption of selected amino acids at mercury/aqueous solution of chlorate (VII) interface; dependence on the supporting electrolyte concentration. Adsorpt Sci. Technol. 2015;33:553-8.

9. Ostolska I, Wiśniewska M. Investigation of the colloidal $\mathrm{Cr}_{2} \mathrm{O}_{3}$ removal possibilities from aqueous solution using the ionic polyamino acid block copolymers. J Hazard Mater. 2015;290:69-77.

10. Bagreev A, Menendez JA, Dukano I, Tarasenko Y, Bandosz TJ. Bituminous coal-based activated carbons modified with nitrogen as adsorbents of hydrogen sulfide. Carbon. 2004;42:469-76.

11. Nowicki P, Kazmierczak J, Sawicka K, Pietrzak R. Nitrogenenriched activated carbons prepared by the activation of coniferous tree sawdust and their application in the removal of nitrogen dioxide. Int J Environ Sci Technol. 2015;12:2233-44. 
12. Vargas DP, Giraldo L, Erto A, Moreno-Piraján JC. Chemical modification of activated carbon monoliths for $\mathrm{CO}_{2}$ adsorption. J Therm Anal Calorim. 2013;114:1039-47.

13. Veksha A, Sasaoka E, Uddin MA. The influence of porosity and surface oxygen groups of peat-based activated carbons on benzene adsorption from dry and humid air. Carbon. 2009;47:2371-8.

14. Houshmand A, Daud WMAW, Shafeeyan MS. Exploring potential methods for anchoring amine groups on the surface of activated carbon for $\mathrm{CO}_{2}$ adsorption separation. Sci Technol. 2011;46:1098-112.

15. Foo KY, Hameed BH. Microwave assisted preparation of activated carbon from pomelo skin for the removal of anionic and cationic dyes. Chem Eng J. 2011;173:385-90.

16. Kazmierczak-Razna J, Pietrzak R. The use of microwave radiation for obtaining carbonaceous adsorbents from biomass and their use in elimination of inorganic pollutants. Adsorption. 2015;. doi:10.1007/s10450-015-9713-5.

17. Rechnia P, Malaika A, Krzyżyńska B, Kozłowski M. Decomposition of methane in the presence of ethanol over activated carbon catalyst. Int J Hydrog Energy. 2012;37(19):14178-86.

18. Rechnia P, Malaika A, Kozłowski M. Synthesis of tert-amyl methyl ether (TAME) over modified activated carbon catalysts. Fuel. 2015;154:338-45.

19. Malaika A, Kozłowski M. Modification of activated carbon with different agents and catalytic performance of products obtained in the process of ethylbenzene dehydrogenation coupled with nitrobenzene hydrogenation. Chem Eng J. 2011;171(3):1348-55.

20. Jurewicz K, Pietrzak R, Nowicki P, Wachowska H. Capacitance behaviour of brown coal based active carbon modified through chemical reaction with urea. Electrochim Acta. 2008;53:5469-75.

21. Pietrzak R, Jurewicz K, Nowicki P, Babeł K, Wachowska H. Nitrogen-enriched bituminous coal based active carbons as materials for supercapacitors. Fuel. 2010;89:3457-67.

22. Wachowski L, Hofman M. Thermogravimetric and textural studies of modified carbonaceous materials. Thermochim Acta. 2005;437:82-6.

23. Boudou JP, Chehimi M, Broniek E, Siemieniewska T, Bimer J. Adsorption of $\mathrm{H}_{2} \mathrm{~S}$ or $\mathrm{SO}_{2}$ on an activated carbon cloth modified by ammonia treatment. Carbon. 2003;43:1999-2007.

24. Bashkova S, Bandosz TJ. The effects of urea modification and heat treatment on the process of $\mathrm{NO}_{2}$ removal by wood-based activated carbon. J Colloid Interface Sci. 2009;333:97-103.
25. Kazmierczak-Razna J, Nowicki P, Pietrzak R. The use of microwave radiation for obtaining activated carbons enriched in nitrogen. Powder Technol. 2015;273:71-5.

26. Garcia P, Espinal JF, de Lecea CSM, Mondragon F. Experimental characterization and molecular simulation of nitrogen complexes formed upon $\mathrm{NO}-$ char reaction at $270{ }^{\circ} \mathrm{C}$ in the presence of $\mathrm{H}_{2} \mathrm{O}$ and $\mathrm{O}_{2}$. Carbon. 2004;42:1507-15.

27. Bimer J, Sałbut PT, Berłożecki S, Boudou JP, Broniek E, Siemieniewska T. Modified active carbons from precursors enriched with nitrogen functions: sulfur removal capabilities. Fuel. 1998;77(6):519-25.

28. Machnikowski J, Grzyb B, Weber JV, Frackowiak E, Rouzaud JN, Béguin F. Structural and electrochemical characterisation of nitrogen enriched carbons produced by the co-pyrolysis of coaltar pitch with polyacrylonitrile. Electrochim Acta. 2004;49(3):423-32.

29. Boudou JP, Parent P, Suarez-Garcia F, Vilar-Rodil S, MartinezAlonso A, Tascon JMD. Nitrogen in aramid-based activated carbon fibers by TPD, XPS and XANES. Carbon. 2006;44:2452-62.

30. Pollak E, Salitra G, Soffer A, Aurbach D. On the reaction of oxygen with nitrogen-containing and nitrogen-free carbons. Carbon. 2006;44:3302-7.

31. Thote JA, Iyer KS, Chatti R, Labhsetwar NK, Biniwale RB, Rayalu SS. In situ nitrogen enriched carbon for carbon dioxide capture. Carbon. 2010;48:396-402.

32. Plaza MG, Pevida C, Arenillas A, Rubiera F, Pis JJ. $\mathrm{CO}_{2}$ capture by adsorption with nitrogen enriched carbons. Fuel. 2007;86:2204-12.

33. Tamai H, Shiraki K, Shiono T, Yasuda H. Surface functionalization of mesoporous and microporous activated carbons by immobilization of diamine. J Colloid Interface Sci. 2006;295:299-302.

34. Chen W-C, Wen T-C, Teng H. Polyaniline-deposited porous carbon electrode for supercapacitor. Electrochim Acta. 2003;48:641-9.

35. Pietrzak R, Wachowska H, Nowicki P. Preparation of nitrogenenriched activated carbons from brown coal. Energy Fuels. 2006;20:1275-80.

36. Nowicki P, Pietrzak R, Wachowska H. X-ray photoelectron spectroscopy study of nitrogen-enriched active carbons obtained by ammoxidation and chemical activation of brown and bituminous coals. Energy Fuels. 2010;24:1197-206. 